\title{
THE INVERSION OF A TRANSFORM RELATED TO THE LAPLACE TRANSFORM AND TO HEAT GONDUGTION *
}

\author{
DAVID V. WIDDER \\ (received 26 November, 1962)
}

\section{Introduction}

In a recent paper [7] the author considered, among other things, the integral transform

$$
f(t)=\int_{0}^{\infty} k(y, t) \phi(y) d y
$$

where

$$
k(x, t)=(4 \pi t)-t e^{-\sigma^{2} /(4 t)}, \quad t>0,
$$

is the fundamental solution of the heat equation

$$
\frac{\partial^{2} u}{\partial x^{2}}=\frac{\partial u}{\partial t}
$$

There we gave a physical interpretation of the transform (1.1). Here we shall choose a slightly different interpretation, more convenient for our present purposes. If

$$
u(x, t)=\frac{1}{2} \int_{0}^{\infty}[k(x+y, t)+k(x-y, t)] \phi(y) d y,
$$

then $u(0, t)=f(t)$. That is, the function $f(t)$ defined by equation (1.1) is the temperature at the origin $(x=0)$ of an infinite bar along the $x$-axis $t$ seconds after it was at a temperature defined by the equation

$$
u(|x|, 0)=\phi(x), \quad-\infty<x<\infty .
$$

Alternatively, it is clear that (1.1) is also related to the Laplace transform. In fact

$$
t^{-\frac{1}{2}} f(1 / t)=\int_{0}^{\infty} e^{-t y}(4 \pi y)^{-\frac{1}{2}} \phi(\sqrt{4 y}) d y .
$$

It is not surprising then that the transform should have inversion formulas

* This work was supported by the Air Force Office of Scientific Research, under Contract AF $49(638)-574$. 
of two distinct types deriving from the separate theories. We shall develop them here, limiting ourselves to real-valued inversion operators involving the successive derivatives of $f(t)$. (See Corollary 3.2, p. 180, of [6] and Chapters 7 and 8 of [5] for analogous formulas.) The one deriving from heat conduction theory is

$$
\phi(x)=\lim _{t \rightarrow 0+} 2 \sum_{n=0}^{\infty} \frac{x^{2 n}}{(2 n) !} f^{(n)}(t)
$$

Conditions for the validity of (1.4) appear in Theorem 3.1. To describe the second inversion conveniently we first define recursively the following linear differential operators

$$
\begin{aligned}
& L_{0}[f(t)]=f(t) \\
& L_{1}[f(t)]=t^{2} D\left(L_{0}[f(t)]\right) \\
& \cdots \\
& L_{n}[f(t)]=t^{2} D\left(L_{n-1}[f(t)]\right), \quad n=1,2,3, \cdots .
\end{aligned}
$$

Here the symbol $D$ stands for differentiation with respect to $t$. If the $n$ differentiations indicated in (1.5) are performed, the equation takes the familiar Euler form (p. 76 of [2]), except for an inconsequential factor $t^{n}$ :

$$
L_{n}[f(t)]=t^{n} \sum_{k=0}^{n} a_{k} t^{k} f^{(k)}(t), \quad a_{n}=1 .
$$

And now the second inversion is (Theorem 4.1 below)

$$
\lim _{n \rightarrow \infty} \frac{\sqrt{\pi}}{n !} \frac{(4 n)^{n+1}}{y^{2 n+1}}\left\{L_{n}[\sqrt{t} f(t)]\right\}_{t=y^{2 /(4 n)}}=\phi(y), \quad 0<y<\infty .
$$

Finally, an analogue of S. Bernstein's representation theorem for the Laplace transform (p. 161 of [5]), here applicable to (1.1), is obtained. It is shown that a function $f(t)$ has a representation by the Stieltjes integral

$$
f(t)=\int_{0}^{\infty} k(y, t) d \alpha(y), \quad 0<t<\rho,
$$

with $\propto(y)$ non-decreasing, if and only if $L_{n}\left[t \frac{1}{2} f(t)\right] \geqq 0, n=0,1,2, \cdots$ on the interval $0<t<\rho$. A simple illustration is provided by taking $f(t)=\sqrt{t / \pi}, \alpha(y)=y^{2} / 2$ (see p. 283 of $[7]$ ). Then for $n=0,1,2, \cdots$

$$
L_{n}[\sqrt{t} f(t)]=n ! t^{n+1} / \sqrt{\pi} \geqq 0, \quad 0<t<\infty .
$$

This example also gives an illustration of formula (1.6) with $\phi(y)=\alpha^{\prime}(y)=y$. The left hand side of equation (1.6) reduces to $y$ (even without the limit operation). 
By applying (1.6) to (1.3) we obtain for the Laplace transform an inversion which appears to be new. See Theorem 6.1 below.

\section{Region of convergence}

Equation (1.3) enables us to obtain the basic properties of the transform (1.1). Let us consider the more general Stieltjes integral form

$$
f(t)=\int_{0}^{\infty} k(y, t) d \alpha(y) .
$$

Here, and subsequently, $\alpha(y)$ is a function of bounded variation in $0 \leqq y \leqq R$ for every positive $R$, and $\alpha(0)=0$. The transform is defined for real $t>0$, but of course it can be continued analytically into the complex $t$-plane, $t=t_{1}+i t_{2}$. We shall obtain the region of convergence in this plane, although the transform will be considered real throughout the remainder of the paper.

If $1 / t=v=v_{1}+i v_{2}$, a simple change of variable gives

$$
v^{\frac{1}{2}} f(1 / v)=(4 \pi)^{-\frac{1}{2}} \int_{0}^{\infty} e^{-v v} d \alpha(2 \sqrt{y}) .
$$

If (2.1) converges for some real $t>0$, then (2.2) will converge for some real $v>0$, and since the region of convergence of the Laplace integral (2.2) in the complex $v$-plane is the half-plane $v_{1}>1 / \rho \geqq 0$, it follows that the region of convergence of $(2.1)$ in the complex $t$-plane is $t_{1} /\left(t_{1}^{2}+t_{2}^{2}\right)>1 / p$. That is, it is the interior of a circle through the origin and with diameter the interval of the real axis $0<t_{1}<\rho$. Known basic properties of the Laplace transform give us the following result.

THEOREM 2.1. If the real transform (2.1) converges for some real $t>0$, then it converges in an interval $0<t<\rho$ and diverges for $t>\rho$. The function $f(t)$ is analytic on that interval, and its successive derivatives may be obtained by formal differentiation under the integral sign. It is the restriction to the real axis of a function analytic in a circle of the complex t-plane rohose diameter is the real interval $(0, \rho)$. The limiting case $\rho=\infty$ is admissible.

The Stieltjes integral (2.1) of course includes the Lebesgue form (1.1) when $\alpha(y)$ is absolutely continuous. However (2.1) can always be reduced to the form (1.1) at the expense of a factor $1 /(2 t)$. If $(2.1)$ converges for $0<t<\rho$, we have by Theorem 2.2a, p. 39, of [5] that

$$
\alpha(y)=o\left(e^{\nu^{2} /(4 \rho \theta)}\right),
$$$$
y \rightarrow \infty \text {, }
$$

for any positive number $\theta<1$. Applying integration by parts to the integral (2.1), we have

$$
f(t)=\left.k(y, t) \alpha(y)\right|_{0} ^{\infty}-\int_{0}^{\infty} \frac{\partial}{\partial y} k(y, t) \alpha(y) d y .
$$


By (2.3) and the assumption $\alpha(0)=0$, the integrated term disappears. We thus obtain

$$
f(t)=\frac{1}{2 t} \int_{0}^{\infty} k(y, t) y \alpha(y) d y, \quad 0<t<\rho .
$$

\section{Inversion. Heat type}

We are now in a position to prove the following result.

THEOREM 3.1. If $\phi(y) \in L$ on $0 \leqq y \leqq R$ for every $R>0$, and if

$$
f(t)=\int_{0}^{\infty} k(y, t) \phi(y) d y,
$$

the integral converging absolutely for $0<t<\rho$, then

$$
\phi(y)=\lim _{t \rightarrow 0+} 2 \sum_{n=0}^{\infty} \frac{y^{9 n}}{(2 n) !} f^{(n)}(t)
$$

for almost all positive $y$. If for some $y_{0}>0$ the limits $\phi\left(y_{0}+\right)$ and $\phi\left(y_{0}-\right)$ exist, then the limit (3.2) is equal to $\left[\phi\left(y_{0}+\right)+\phi\left(y_{0}-\right)\right] / 2$.

Consider the integral (1.2), the function $\phi(y)$ of the integrand having the properties assumed in the present theorem. By comparison with (3.1) it converges absolutely for $0<t<\rho,-\infty<x<\infty$ and thus defines a function $u(x, t)$ in that strip. Expand the integrand in powers of $x$ by Taylor's series

$$
\begin{aligned}
u(x, t) & =\int_{0}^{\infty} \phi(y) \sum_{n=0}^{\infty} \frac{x^{2 n}}{(2 n) !} \frac{\partial^{2 n}}{\partial y^{2 n}} k(y, t) d y \\
& =\sum_{n=0}^{\infty} \frac{x^{2 n}}{(2 n) !} \frac{\partial^{n}}{\partial t^{n}} \int_{0}^{\infty} k(y, t) \phi(y) d y \\
& =\sum_{n=0}^{\infty} \frac{x^{2 n}}{(2 n) !} f^{(n)}(t) .
\end{aligned}
$$

Here we have used Theorem 2.1 to validate differentiation under the integral sign. The term-by-term integration of the series will be valid if

$$
\sum_{n=0}^{\infty} \frac{x^{2 n}}{(2 n) !} \int_{0}^{\infty}|\phi(y)|\left|\frac{\partial^{2 n}}{\partial y^{2 n}} k(y, t)\right| d y<\infty
$$

By equation (2.4) of [3]

$$
w_{n}(y, t)=(-2)^{n} \frac{\partial^{n}}{\partial y^{n}} k(y, t) .
$$

For present purposes this equation may be taken as a definition. By equation 
(3.5) of [3]

$$
\left|w_{n}(y, t)\right| \leqq A e^{-y^{2} / 8 t}\left(\frac{2 n}{e t}\right)^{n / 2} \frac{n t}{t !},
$$

where $A$ is some constant. Thus (3.3) will hold when

$$
\begin{aligned}
& \sum_{n=0}^{\infty} \frac{x^{2 n}}{(2 n) !}\left(\frac{n}{e t}\right)^{n}(2 n) t<\infty \\
& \int_{0}^{\infty} e^{-v^{2} / 8 t}|\phi(y)| d y<\infty .
\end{aligned}
$$

Stirling's formula, or the ratio test, shows that (3.5) is true for $-\infty<x<$ $\infty, 0<t<\infty$. By the assumed absolute convergence of (3.1) for $0<t<\rho$, the inequality (3.6) holds for $0<t<\rho / 2$. Hence we have proved that $u(x, t)$ is defined in that strip and that

$$
\begin{aligned}
u(x, t) & =\frac{1}{2} \int_{-\infty}^{\infty} k(x-y, t) \phi(y) d y, \quad \phi(-y)=\phi(y) \\
& =\sum_{n=0}^{\infty} \frac{x^{2 n}}{(2 n) !} f^{(n)}(t) .
\end{aligned}
$$

But (3.7) is the Poisson or Weierstrass integral invaluable in the theory of heat conduction. Our proof can now be completed by reference to Corollaries $7.2 \mathrm{a}$ and $7.2 \mathrm{~b}, \mathrm{p} .189$ of $[6]$.

Although we do not need the result, we observe that the equations (3.7), (3.8) hold in the larger strip $0<t<\rho$. This may be seen by use of analytic continuation. For fixed $x$ the integral (3.7)defines a function analytic for $0<t<\rho$. A slight modification of the proof used for Theorem 2.1 would show this. Moreover, for fixed $x$ the sum of the series (3.8) is also analytic for $0<t<\rho$. For, the series converges uniformly in any compact region $S$ of the complex $t$-plane inside the circle of convergence of (1.1). Indeed, by Theorem 2.1, an inequality of the form

$$
\left|f^{(n)}(t)\right| \leqq \frac{M n !}{r^{n}}
$$

holds there; whence, for any fixed $x_{0}$,

$$
\sum_{n=0}^{\infty} \frac{x_{0}^{2 n}}{(2 n) !} f^{(n)}(t) \ll M \sum_{n=0}^{\infty} \frac{x_{0}^{2 n}}{(2 n) !} \frac{n !}{r^{n}}<\infty, \quad t \in S .
$$

Consequently the equality (3.7) to $(3.8)$, proved above for $0<t<\rho / 2$, must also hold for $0<t<\rho$.

We turn next to the Stieltjes case and prove 
THEOREM 3.2. If

$$
f(t)=\int_{0}^{\infty} k(y, t) d \alpha(y),
$$

the integral converging for $0<t<\rho$, then

$$
\lim _{t \rightarrow 0+} 2 \sum_{n=0}^{\infty} \frac{y^{2 n+1}}{(2 n+1) !} f^{(n)}(t)= \begin{cases}{[\alpha(y+)+\alpha(y-)] / 2,} & y>0 \\ \alpha(0)=0, & y=0 .\end{cases}
$$

We begin by an integration by parts, obtaining the equation (2.4). By virtue of the order relation (2.3), the integral (2.4) converges absolutely for $0<t<\rho$. Hence Theorem 3.1 is applicable to the function $t f(t)$. We are thus led to consider the series

$$
\begin{aligned}
& \sum_{n=0}^{\infty} \frac{y^{2 n}}{(2 n) !} \frac{d^{n}}{d t^{n}}(t f(t))= \\
& t \sum_{n=0}^{\infty} \frac{y^{2 n}}{(2 n) !} f^{(n)}(t)+\frac{y}{2} \sum_{n=0}^{\infty} \frac{y^{2 n+1}}{(2 n+1) !} f^{(n)}(t) .
\end{aligned}
$$

Here we have differentiated $t f(t) n$ times and increased the dummy variable $n$ by 1 in the second sum. By Theorem 3.1 applied to (2.4) the sum of the series (3.11) tends to $y \alpha(y) / 4$, at least at points of continuity of $\alpha(y)$. Since $\alpha(y)$ is assumed to be of bounded variation in every finite interval, $\alpha(y+)$ and $\alpha(y-)$ exist for $y>0$. Hence equation (3.10) will be established if we show that the sum of the first series (3.12) tends to zero as $t \rightarrow 0+$. From (2.4) we have

$$
f^{(n)}(t)=-\int_{0}^{\infty} \frac{\partial^{2 n+1}}{\partial y^{2 n+1}} k(y, t) \alpha(y) d y .
$$

By use of Maclaurin's series we have

$$
\begin{aligned}
\sum_{n=0}^{\infty} \frac{x^{2 n}}{(2 n) !} f^{(n)}(t) & =-\frac{1}{2} \int_{0}^{\infty}\left[k_{1}(x+y, t)-k_{1}(x-y, t)\right] \alpha(y) d y \\
& =-\frac{1}{2} \int_{-\infty}^{\infty} k_{1}(x-y, t) \alpha(y) d y, \quad \alpha(-y)=\alpha(y) .
\end{aligned}
$$

Here $k_{1}(y, t)$ is the partial derivative of $k(y, t)$ with respect to $y$. As in the proof of Theorem 3.1 we have expanded the integrand (3.13) in powers of $x$ and integrated the resulting series term by term. This process is valid if

$$
\sum_{n=0}^{\infty} \frac{(x / 2)^{2 n}}{(2 n) !} \int_{0}^{\infty}\left|\alpha(y) \| w_{2 n+1}(y, t)\right| d y<\infty .
$$

Again using the inequality (3.4) we see that this holds for $0<t<\rho / 2$. 
In that strip we have accordingly proved that

$$
t \sum_{n=0}^{\infty} \frac{x^{2 n}}{(2 n) !} f^{(n)}(t)=\frac{1}{4} \int_{-\infty}^{\infty} k(x-y, t)(x-y) \alpha(y) d y .
$$

But as $t \rightarrow 0+$ this Poisson integral approaches 0 , the value of $(x-y) \alpha(y)$ when $y=x$. This completes the proof of the theorem.

\section{Inversion. Laplace integral type}

Let us first prove a simple identity involving the linear differential operators $L_{n}$ defined by (1.5).

Lemma 4.1. If $f(t) \in C^{n}$ on $0<t<\rho$ and $n=0,1,2, \cdots$, then

$$
L_{n}[t(t)]=\left.(-1)^{n} \frac{d^{n}}{d x^{n}} f\left(\frac{1}{x}\right)\right|_{x=1 / t}, \quad 0<t<\rho .
$$

This identity can be proved by direct calculation or by induction. Perhaps the simplest proof consists in noting that both sides of (4.1) involve linear differential operators of order $n$ with leading term $t^{2 n} f^{(n)}(t)$. Moreover, for $n>0$ each operator annuls the same set of $n$ linearly independent functions:

$$
1, t^{-1}, t^{-2}, \cdots, t^{-n+1} \text {. }
$$

The identity, obvious for $n=0$, is thus established.

THEOREM 4.1. If $\phi(y) \in L$ on $0 \leqq y \leqq R$ for every $R>0$, and if

$$
f(t)=\int_{0}^{\infty} k(y, t) \phi(y) d y
$$

the integral converging for $0<t<\rho$, then

$$
\lim _{n \rightarrow \infty} \frac{\sqrt{\pi}}{n !} \frac{(4 n)^{n+1}}{y^{2 n+1}}\left\{L_{n}\left[t \frac{1}{t} f(t)\right]\right\}_{t \rightarrow y^{3} /(4 n)}=\phi(y)
$$

for almost all positive $y$. If for some $y_{0}>0$ the limits $\phi\left(y_{0}+\right)$ and $\phi\left(y_{0}-\right)$ exist, then the limit (4.3) is $\left[\phi\left(y_{0}+\right)+\phi\left(y_{0}-\right)\right] / 2$.

Note that in contrast with Theorem 3.1 we do not here assume the absolute convergence of the integral (4.2). To prove the theorem we observe first that

$$
t^{-\frac{1}{2}} L_{n}\left[t \frac{1}{2} k(x, t)\right]=\left(\frac{x}{2}\right)^{2 n} k(x, t),
$$

Hence by Theorem 2.1

$$
t^{-\frac{1}{2}} L_{n}\left[t^{\frac{t}{2}} f(t)\right]=\int_{0}^{\infty} k(x, t)\left(\frac{x}{2}\right)^{2 n} \phi(x) d x
$$


That is, the left hand side of $(4.3)$ is

$$
\begin{aligned}
& \lim _{n \rightarrow \infty} \frac{2}{n !} \frac{n^{n+1}}{y^{2 n+1}} \int_{0}^{\infty} e^{-n x^{2} / y^{2}} x^{2 n} \phi(x) d x= \\
& \lim _{n \rightarrow \infty} \frac{y}{n !}\left(\frac{n}{y^{2}}\right)^{n+1} \int_{0}^{\infty} e^{-n x / y^{2}} x^{n-1} \phi\left(x^{\frac{1}{1}}\right) d x .
\end{aligned}
$$

But the value of this limit is known (Corollaries 3c.1 and 3c.2, p. 283 of [5]), and accords with the conclusions of the theorem.

An alternative proof by use of the identities (1.3) and (4.1) is also available. For, by (4.1)

$$
\left.L_{n}[f(t) \sqrt{ } t]\right|_{t=1 / x}=(-1)^{n} \frac{d^{n}}{d x^{n}}\left[f\left(\frac{1}{x}\right)\left(\frac{1}{x}\right)^{\frac{1}{t}}\right] .
$$

But

$$
\lim _{n \rightarrow \infty} \frac{(-1)^{n}}{n !}\left(\frac{n}{y}\right)^{n+1} \frac{d^{n}}{d x^{n}}\left[f\left(\frac{1}{x}\right)\left(\frac{1}{x}\right)^{\frac{1}{2}}\right]_{x=n / y}
$$

is the known inversion operator for the Laplace integral (1.3). (See p. 288 of [5]). Hence the latter limit is the determining function of (1.3), or $(4 \pi y)^{-\frac{1}{1}} \phi(\sqrt{4 y})$. To obtain $\phi(y)$ as a limit we should replace $x$ by $4 n / y^{2}$ in (4.5). Thus

$$
\lim _{n \rightarrow \infty} \frac{1}{n !}\left(\frac{4 n}{y^{2}}\right)^{n+1}\left\{L_{n}[f(t) \sqrt{ } t]\right\}_{t-y^{2} /(4 n)}=\frac{\phi(y)}{y \sqrt{ } \pi},
$$

at least at points where $\phi(y)$ is continuous. The conclusions of the theorem are now immediate.

For the Stieltjes integral (3.9) we have

THEOREM 4.2. Under the hypotheses of Theorem 3.2

$$
\lim _{n \rightarrow \infty} \frac{2 \sqrt{ } \pi}{n !}\left\{\frac{1}{t^{n+1}} L_{n}\left[t^{2} f(t)\right]\right\}_{t=y^{2} / 4 n}=\frac{\alpha(y+)+\alpha(y-)}{2}
$$

for all $y>0$.

The proof is made by applying Theorem 4.1 to (2.4). We omit the details.

\section{A representation result}

The positiveness of $L_{n}[f(t)]$ for all $n$ guarantees that $f(t)$ has the integral representation (2.1) with $\alpha(y)$ increasing.

THEOREM 5.1. A necessary and sufficient condition that

$$
f(t)=\int_{0}^{\infty} k(y, t) d \alpha(y)
$$$$
0<t<\rho,
$$ 
with $\alpha(y)$ non-decreasing is that $L_{n}\left[t \frac{1}{1} f(t)\right] \geqq 0$ on $0<t<\rho$ for $n=0,1,2, \cdots$.

The necessity of the condition follows from equation (4.4). Conversely, from Lemma 4.1 we see that $L_{n}\left[t \frac{1}{t} f(t)\right] \geqq 0$ implies that $x^{-\frac{1}{2}} f(1 / x)$ is completely monotonic on $1 / \rho<x<\infty$. By Bernstein's theorem referred to above

$$
x^{-\frac{1}{2}} f(1 / x)=\int_{0}^{\infty} e^{-x y} d \beta(y), \quad 1 / \rho<x<\infty,
$$

where $\beta(y)$ is non-decreasing. The proof is now completed by setting $x=1 / t$ and $\alpha(y)=2 \pi \frac{1}{2} \beta\left(y^{2} / 4\right)$.

There is a very simple and elegant sufficient condition that $f(t)$ should have the representation (5.1) or (1.1). It is that $f(t)$ should be analytic at $t=0$. Of course the condition is very far from being necessary. This may be seen by consideration of equation (1.2). Solutions of the heat equation cannot generally be extended backwards in time. Or, from the following theorem it will be seen that the analyticity of $f(t)$ at $t=0$ implies that of $\phi(y)$ for $-\infty<y<\infty$. On the other hand the transform (1.1) may be well defined for discontinuous functions $\phi(y)$, for example.

THEOREM 5.2. If $f(t)$ is analytic at $t=0$,

$$
f(t)=\sum_{n=0}^{\infty} f^{(n)}(0) \frac{t^{n}}{n !}, \quad|t|<\rho,
$$

then

$$
f(t)=\int_{0}^{\infty} k(y, t) \phi(y) d y, \quad 0<t<\rho,
$$

where $\phi(y)$ is an even entire function of growth $(2,1 / 4 \rho)$,

$$
\phi(y)=2 \sum_{n=0}^{\infty} \frac{y^{2 n}}{(2 n) !} f^{(n)}(0), \quad-\infty<y<\infty
$$

From hypothesis (5.2) we have

$$
\limsup _{n \rightarrow \infty} n^{-1}\left|f^{(n)}(0)\right|^{1 / n} \leqq(e p)^{-1} .
$$

A function $\sum_{n=0}^{\infty} c_{n} z^{n} / n !$ is of growth $(2, \sigma)$ if and only if

$$
\limsup _{n \rightarrow \infty} n^{-1}\left|c_{n}\right|^{2 / n} \leqq 2 \sigma e^{-1} \text {. }
$$

(See, for example, p. 11 of [1]). Taking into account that $\phi(y)$, as defined by (5.4), is an even function, we see that (5.5) is precisely the condition that $\phi(y)$ should be entire of growth $(2,1 / 4 \rho)$.

Now substitute series (5.4) in integral (5.3) and use the identity 
(p. 283 of $[7]$ )

$$
\frac{t^{n}}{n !}=2 \int_{0}^{\infty} k(y, t) \frac{y^{2 n}}{(2 n) !} d y .
$$

Thus, $f(t)$ is equal to the sum of the series (5.2) if term-by-term integration is valid. For this it is sufficient that

or that

$$
\sum_{n=0}^{\infty}\left|f^{(n)}(0)\right| \int_{0}^{\infty} k(y, t) \frac{y^{2 n}}{(2 n) !} d y<\infty,
$$

$$
\sum_{0}^{\infty}\left|f^{(n)}(0)\right| \frac{t^{n}}{n !}<\infty
$$

Since the latter inequality follows from (5.2) the proof is complete.

: Note that the inversion provided by equation (5.4) is a special case of (3.2) in which the limit symbol may be transferred under the summation symbol. This transfer, of course, is not generally permissible, as the example $f(t)=\sqrt{t / \pi}$ of $\S 1$ shows.

From equation (5.4) it follows that $\phi^{(2 n)}(0)=2 f^{(n)}(0)$. This is to be expected since $u(x t)$ as defined by (1.2) satisfies the heat equation at the origin under the present hypothesis, and since $u(x, t)$ reduces to $f(t)$ on the $t$-axis and to $\phi(x) / 2$ on the $x$-axis.

\section{Application to the Laplace transform}

Since equations (1.1) and (1.3) are equivalent it is clear that an inversion of the integral (1.1) must also apply to the Laplace integral (1.3). In this way Theorem 3.1 leads to

THEOREM 6.1. If $\phi(y) \in L$ on $0 \leqq y \leqq R$ for every $R>0$, and if

$$
f(x)=\int_{0}^{\infty} e^{-x y} \phi(y) d y,
$$

the integral converging absolutely for $x>1 / p \geqq 0$, then

$$
\phi(y)=\lim _{x \rightarrow \infty} \frac{1}{\sqrt{\pi y}} \sum_{n=0}^{\infty} \frac{(-4 y)^{n}}{(2 n) !} L_{n}\left[x^{\frac{1}{2}} f(x)\right]
$$

for almost all positive $y$. If for $y_{0}>0$ the limits $\phi\left(y_{0}+\right)$ and $\phi\left(y_{0}-\right)$ exist then the limit $(6.2)$ is $\left[\phi\left(y_{0}+\right)+\phi\left(y_{0}-\right)\right] / 2$.

For, (6.1) is equivalent to

$$
t^{-\frac{1}{2}} f(1 / t)=\sqrt{ } \pi \int_{0}^{\infty} k(y, t) \phi\left(y^{2} / 4\right) y d y .
$$


Hence (3.2) becomes

$$
\sqrt{\pi} \phi\left(y^{2} / 4\right) y=\lim _{t \rightarrow 0+} 2 \sum_{n=0}^{\infty} \frac{y^{2 n}}{(2 n) !} \frac{d^{n}}{d t^{n}}\left[\frac{1}{\sqrt{ } t} f\left(\frac{1}{t}\right)\right] .
$$

Finally, (6.2) results from Lemma 4.1 and obvious changes of variable. Note that formula (6.2) may well be very simply applied to certain special functions when standard inversions are more complicated. For example, if $f(x)=x^{-1}, L_{n}\left[x^{t} f(x)\right]=0$ for $n \geqq 1$. Hence series (6.1) reduces to its first term, and $\phi=(\pi y)^{-1}$.

The corresponding result for the Stieltjes integral is

THEOREM 6.2. If

$$
f(x)=\int_{0}^{\infty} e^{-x y} d \alpha(y),
$$

the integral converging for $x>1 / \rho \geqq 0$, then

$$
\lim _{x \rightarrow \infty} 2\left(\frac{y}{\pi}\right)^{\frac{1}{2}} \sum_{n=0}^{\infty} \frac{(-4 y)^{n}}{(2 n+1) !} L_{n}\left[x^{\frac{1}{t}} f(x)\right]=\frac{\alpha(y+)+\alpha(y-)}{2}, y>0 .
$$

This result is essentially a corollary of Theorem 3.2. As an example, if $f(x)=1, L_{n}\left[x^{\frac{1}{2}}\right]=(2 n) !(x / 4)^{n} \sqrt{x} / n !$, and $(6.3)$ becomes

$$
\lim _{x \rightarrow \infty} \operatorname{erf} \sqrt{x y}=1, \quad y>0 .
$$

Thus $\alpha(y)$ is a step-function with unit jump at the origin.

\section{Examples}

A simple transform (1.1) results if $\phi(y)=2 \cosh a y$. Then $f(t)=\exp a^{2} t$. Equation (3.2) becomes

Equation (3.10) becomes

$$
\phi(y)=2 \cosh a y=\lim _{t \rightarrow 0+} 2 e^{a^{2} t} \cosh a y .
$$

$$
\alpha(y)=2 a^{-1} \sinh a y=\lim _{t \rightarrow 0+} 2 a^{-1} e^{a^{2} t} \sinh a y .
$$

Thus, $\alpha^{\prime}(y)=\phi(y)$ in this case. This example also gives a trivial illustration of Theorem 5.2. For, $\phi(y)$ is entire of order 1 and hence is of growth $(2, \sigma)$ for any $\sigma$, however small. This behavior of $\phi(y)$ is reflected in the fact that $f(t)$ is now entire. Since $\alpha(y)$ is increasing, Theorem 5.1 indicates that $L_{n}[t z f(t)] \geqq 0$. This fact can be seen directly by use of $(4.1)$. The function $x^{-\frac{1}{2}} \exp a^{2} x^{-1}$, is completely monotonic since it is the product of two such functions. 
Let us now use the example $f(t)=(t / \pi) t, \phi(y)=y$, used in $\S 1$, as an illustration of Theorem 3.1. The series (3.2) becomes

$$
2\left(\frac{t}{\pi}\right)^{t}+2 \sum_{n=0}^{\infty} \frac{(-1)^{n}}{(4 \pi t)^{\frac{1}{2}}} \frac{y^{2 n+2}}{(2 n+2)(2 n+1)} \frac{1}{n !(4 t)^{n}}=4 t k(y, t)+y \operatorname{erf} y(4 t)-t .
$$

This function does tend to $y$ as $t$ approaches $0+$, as predicted.

Finally let us use Theorem 5.2 to obtain the image of the function $f(t)=1 /(1-t)$, analytic for $|t|<1$. From (5.4) it is

$$
\phi(y)=2 \sum_{n=0}^{\infty} \frac{n !}{(2 n) !} y^{2 n} .
$$

This function may be expressed in terms of the error function as follows:

$$
\phi(y)=2+\sqrt{\pi} y e^{v^{2} / 4} \operatorname{erf}(y / 2) .
$$

This may be proved by multiplication of power series or checked by use of formula 22 on p. 235 of [4]. Since $\rho=1, \phi(y)$ should have growth $(2,1 / 4)$, and this is evident from (7.1).

\section{An alternative inversion formula}

The function

$$
u(x, t)=2 \sum_{n=0}^{\infty} \frac{x^{2 n}}{(2 n) !} f^{(n)}(t)
$$

used in the inversion formula (3.2) is formally a solution of the heat equation. If we denote by $D$ the operation of differentiation with respect to $t$, equation (8.1) may be written symbolically as

$$
u(x, t)=2(\cosh x \sqrt{ } D) f(t) .
$$

Formal differentiation gives

$$
u_{\text {ex }}=u_{t}=2(\cosh x \sqrt{ } D) f^{\prime}(t) .
$$

Since the function $2(\sinh x \sqrt{ } D) f(t)$ is also a solution of the heat equation we might expect to find an inversion of (1.1) based thereon. In fact this is possible. Write

$$
2 \sinh x \sqrt{ } D=2\left(\sum_{n=0}^{\infty} \frac{x^{2 n+1}}{(2 n+1) !} D^{n}\right) \sqrt{ } D
$$

and interpret $\sqrt{ } D$ as the usual fractional derivative

$$
g(t)=\sqrt{ } D f(t)=\frac{1}{\sqrt{ } \pi} D \int_{0}^{t} \frac{f(y)}{\sqrt{t-y}} d y .
$$


These considerations would lead one to predict

THEOREM 8.1. Under the conditions of Theorem 3.1

$$
\phi(y)=\lim _{t \rightarrow 0+} 2 \sum_{n=0}^{\infty} \frac{y^{2 n+1}}{(2 n+1) !} g^{(n)}(t)
$$

for almost all positive $y$. Here $g(t)$ is defined by (8.2).

We omit details of proof. But observe that we have essentially proved the result in Theorem 3.2. It is easy to see that $\sqrt{ } D k(y, t)=-k_{1}(y, t)$, so that

$$
g(t)=1 /(2 t) \int_{0}^{\infty} k(y, t) y \phi(y) d y .
$$

But this is equation (2.4) with $f$ replaced by $g$ and $\alpha$ by $\phi$. Hence equation (3.10) gives the desired result, at least if $\phi(y)$ is of bounded variation and $\phi(y+)=\phi(y-)$. Familiarity with the properties of the Poisson integral (3.14) makes the complete result of the theorem evident.

The presence of a fractional derivative in formula (8.3) may make it seem more complicated than (3.1). For certain special functions $f(t)$ it may in fact be less complicated. For example, take the second illustration of $\S 7$, $f(t)=(t / \pi)^{t}, \phi(y)=y$. Then $g(t)=1 / 2$, so that the series (8.3) reduces to its first term, and the inversion is trivial.

This new inversion enables us to supplement the result of Theorem 5.2. There $\phi(y)$ was even, and it is natural to seek a corresponding result for which $\phi(y)$ is an odd entire function. The result is stated in the following theorem.

THEOREM 8.2. If $f(t) t-1$ is analytic at $t=0$,

$$
f(t)=\sqrt{t} \sum_{n=0}^{\infty} \frac{a_{n} t^{n}}{n !}, \quad|t|<\rho,
$$

then

$$
f(t)=\int_{0}^{\infty} k(y, t) \phi(y) d y, \quad 0<t<\rho,
$$

where $\phi(y)$ is an odd entire function of groveth $(2,1 / 4 \rho)$,

$$
\phi(y)=\sqrt{\pi} \sum_{n=0}^{\infty} \frac{(2 n+1) !}{n ! n !} \frac{a_{n}}{4^{n}} \frac{y^{2 n+1}}{(2 n+1) !}, \quad-\infty<y<\infty .
$$

To prove this, substitute series (8.6) in integral (8.5) and use the identity (p. $283[7]$ )

$$
\int_{0}^{\infty} k(y, t) y^{2 n+1} d y=\frac{4^{n}}{\sqrt{ } \pi} n ! t^{n+\frac{1}{2}}
$$


If

$$
b_{n}=\frac{(2 n+1) !}{n ! n !} \frac{a_{n}}{4^{n}}
$$

it follows from (5.5) that

$$
\limsup _{n \rightarrow \infty} \frac{\left|b_{n}\right|^{1 / n}}{2 n} \leqq \frac{2}{4 \rho e},
$$

and this proves, by use of (5.6) with $n$ replaced by $2 n$, that $\phi(y)$ has growth $(2,1 / 4 \rho)$. Details are like those for the proof of Theorem 5.2.

The inversion of $(8.5)$ provided by $(8.6)$ is a special case of $(8.3)$. For, since

$$
\sqrt{ } D t^{n+\frac{1}{2}}=\frac{\Gamma\left(n+\frac{3}{2}\right)}{n !} t^{n}=\frac{\sqrt{\pi}}{2} \frac{(2 n+1) !}{n ! n !}\left(\frac{t}{4}\right)^{n}
$$

we have

$$
g^{(n)}(0)=\frac{(2 n+1) !}{n ! n !} \frac{\sqrt{\pi}}{2^{2 n+1}} a_{n}
$$

Thus, the limit (8.3) becomes precisely (8.6).

By combining Theorems 5.2 and 8.2 we have an especially simple inversion of (1.1) when $\phi(y)$ is of order 2 (or less) and of finite type.

\section{References}

[1] Boas, R. P., Entire Functions, Academic Press (1954).

[2] Ford, L. R., Differential Equations, McGraw-Hill (1955).

[3] Rosenbloom, P. C. and Widder, D. V., Expansions in terms of heat polynomials and associated functions, Trans. Amer. Math. Soc., 92 (1959), 220-266.

[4] Staff of the Bateman Manuscript Project, Tables of Integral Transforms, vol. 1, McGrawHill (1954).

[5] Widder, D. V., The Laplace Transform, Princeton University Press (1946).

[6] Widder, D. V., The Convolution Transform, Princeton University Press (1955).

[7] Widder, D. V., Integral transforms related to heat conduction, Annali di Matematica, 42 (1956), 279-305.

Harvard University

and University of Melbourne. 